\title{
PageRank for Product Image Search
}

\author{
Yushi Jing ${ }^{1,2}$ \\ yjing@cc.gatech.edu \\ Shumeet Baluja ${ }^{2}$ \\ shumeet@google.com \\ ${ }^{1}$ College Of Computing, Georgia Institute of Technology, Atlanta GA \\ ${ }^{2}$ Google, Inc. 1600 Amphitheater Parkway, Mountain View, CA
}

\begin{abstract}
In this paper, we cast the image-ranking problem into the task of identifying "authority" nodes on an inferred visual similarity graph and propose an algorithm to analyze the visual link structure that can be created among a group of images. Through an iterative procedure based on the PageRank computation, a numerical weight is assigned to each image; this measures its relative importance to the other images being considered. The incorporation of visual signals in this process differs from the majority of largescale commercial-search engines in use today. Commercial search-engines often solely rely on the text clues of the pages in which images are embedded to rank images, and often entirely ignore the content of the images themselves as a ranking signal. To quantify the performance of our approach in a real-world system, we conducted a series of experiments based on the task of retrieving images for 2000 of the most popular products queries. Our experimental results show significant improvement, in terms of user satisfaction and relevancy, in comparison to the most recent Google Image Search results.
\end{abstract}

\section{Categories and Subject Descriptors}

H.3.3 [Information systems]: Information Search and Retrieval; I.4.9 [Computing Methodologies]: Image Processing and Computer Vision

\section{General Terms}

Algorithms

\section{Keywords}

PageRank, Graph Algorithms, Visual Similarity

\section{INTRODUCTION}

Although image search has become a popular feature in many search engines, including Yahoo, MSN, Google, etc., the majority of image searches use little, if any, image information to rank the images. Instead, commonly only the text on the pages in which the image is embedded (text in the body of the page, anchor-text, image name, etc) is used. There are three reasons for this: first, text-based search of web pages is a well studied problem that has achieved a

Copyright is held by the International World Wide Web Conference Committee (IW3C2). Distribution of these papers is limited to classroom use, and personal use by others.

$W W W$ 2008, April 21-25, 2008, Beijing, China.

ACM 978-1-60558-085-2/08/04.

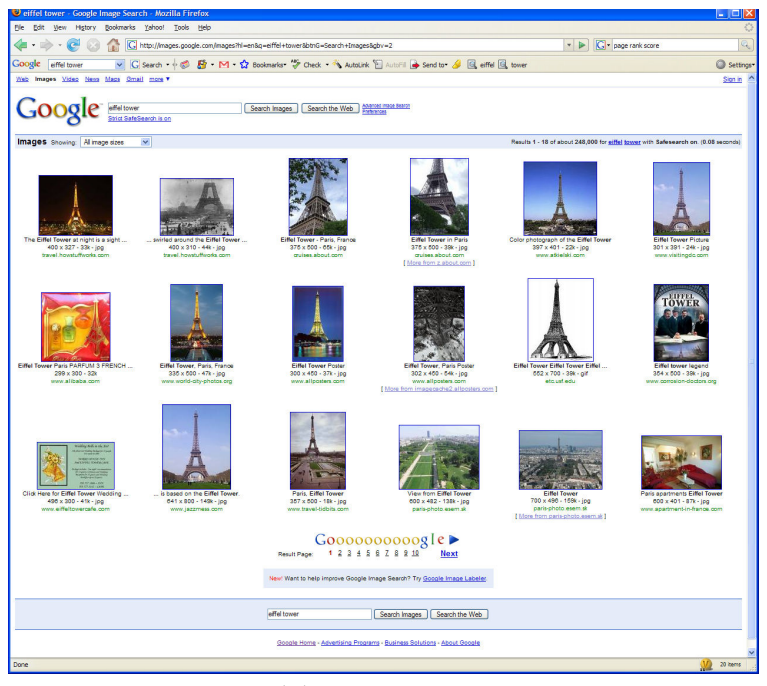

(a) Eiffel Tower

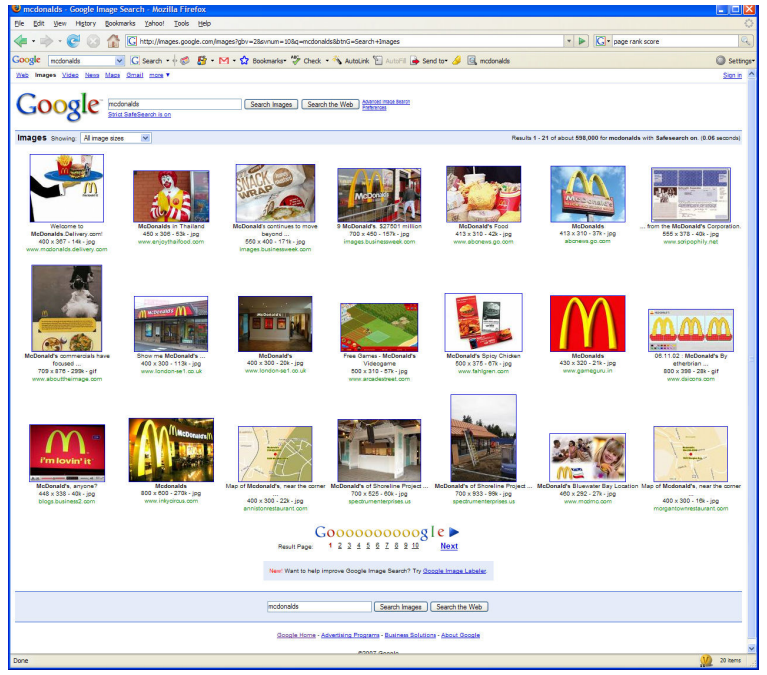

(b) McDonalds.ps

Figure 1: The query for "Eiffel Tower" returns good results on Google. However, the query for "McDonalds" returns mixed results. 
great amount of real-world success. Second, a fundamental task of image analysis is yet largely an unsolved problem: human recognizable objects are usually not automatically detectable in images. Although certain tasks, such as finding faces [17] [15] and highly textured objects like CD covers [12], have been successfully addressed, the problem of general object detection and recognition remains open. Few objects other than those mentioned above can be reliably detected in the majority of images. Third, even for the tasks that are successfully addressed, the processing required can be quite expensive in comparison to analyzing the text of a web-page. Not only do the signal-processing algorithms add an additional level of complexity, but the rapidly increasing average size of images makes the simple task of transferring and analyzing large volumes of data difficult and computationally expensive.

The problem of answering a query without image processing is that it can often yield results that are inconsistent in terms of quality. For example, the query "Eiffel Tower" submitted to image search on Google.com (with strict adult content filtering turned on), returns good results as shown in Figure 1(a). However, the query for "McDonalds" returns mixed results as shown in Figure 1(b); the typical expected yellow "M" logo is not seen as the main component of an image until results 6 and 13 .

The image in Figure 1(b) provides a compelling example of where our approach will significantly improve the image ranking. Our approach relies on analyzing the distribution of visual similarities among the images. The premise is simple: an author of a web page is likely to select images that, from his or her own perspective, are relevant to the topic. Rather than assuming that every user who has a web-page relevant to the query will link to an image that every other user finds relevant, our approach relies on the combined preferences of many web content creators. For example, in Figure 1(b), many of the images contain the familiar "M". In a few of the images, the logo is the main focus of the image, whereas in others it occupies only a small portion. Nonetheless, its repetition in a large fraction of the images is an important signal that can be used to infer a common "visual theme" throughout the set. Finding the multiple visual themes and their relative strengths in a large set of images is the basis of the image ranking system proposed in this study.

There are two main challenges in taking the concept of inferring common visual themes to creating a scalable and effective algorithm. The first challenge is the image processing required. Note that every query may have an entirely separate set of visual features that are common among the returned set. The goal is to find what is common among the images, even though what is common is not a priori known, and the common features may occur anywhere in the image and in any orientation. For example, they may be crooked (Figure 1(b), image 5), rotated out of plane (Figure 1(b), images $4,9,16)$, not be a main component of the image (Figure 1(b), images 1, 8, 20), and even be a non-standard color (Figure 1(b), images 7 and 10). What will make this tractable is that unlike approaches that require analyzing the similarity of images by first recognizing human recognizable objects in the images (i.e. "both these images contain trees and cars"), we do not rely on first detecting objects. Instead, we look for low level features of the images that are invariant to the types of degradations (scale, orientation, etc) that we ex-

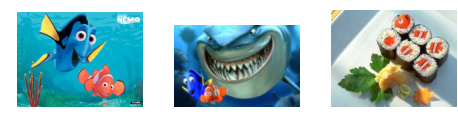

Figure 2: Many queries like "nemo" contain multiple visual themes.

pect to encounter. To address this task, we turn to the use of local features [10] [2]. Mikolajczyk et al. [11] presented a comparative study of various descriptors. Although a full description of local features is beyond the scope of this paper, we provide a brief review in the next section.

The second challenge is that even after we find the common features in the images, we need a mechanism to utilize this information for the purposes of ranking. As will be shown, simply counting the number of common visual features will yield poor results. To address this task, we infer a graph between the images, where images are linked to each other based on their similarity. Once a graph is created, we demonstrate how iterative procedures similar to those used in PageRank can be employed to effectively create a ranking of images. This will be described in Section 2 .

\subsection{Background and Related Work}

There are many methods of incorporating visual signals into search engine rankings. One popular method is to construct an object category model trained from the the top search results, and re-rank images based on their fit to the model [13] [5]. These method obtained promising results, but the assumption of homogeneous object category and limited scale of experiment fall short of offering a conclusive answer on the practicality and performance of such system in commercial search engines. For example, there are significant number of web queries with multiple visual concepts, for example "nemo" (shown in Figure 2). This makes it more difficult to learn a robust model given limited and potentially very diverse set of search results. Further, there is a fundamental mismatch between the goal of object category learning and image ranking. Object category learners are designed to model the relationship between features and images, whereas images search engines are designed to model the relationships (order) among images. Although a well trained object category filter can be used improve the relevancy of image search results, it offers limited capability to directly control how and why one visual theme, or image, is ranked higher than others.

In this work, we propose an intuitive graph-model based method for content-based image ranking. Instead of modelling the relationship between objects and image features, we model the expected user behavior given the visual similarities of the images to be ranked. By treating images as web documents and their similarities as probabilistic visual hyperlinks, we estimate the likelihood of images visited by a user traversing through these visual-hyperlinks. Those with more estimated "visits" will be ranked higher than others. This framework allows us to leverage the well understood PageRank [3] and Centrality Analysis [4] approach for Webpage ranking.

Unlike the web, where related documented are connected by manually defined hyperlinks, we compute visual-hyperlinks explicitly as a function of the visual similarities among images. Since the graph structure will uniquely determine the ranking of the images, our approach offers a layer of abstrac- 
tion from the set of features used to compute the similarity of the image. Similarity can be customized for the types and distributions of images expected; for example, for people queries, facial similarity can be used, color features for landscapes, or local features for architecture, product images, etc.

Several other studies have explored the use of a similarity based graph [8] [19] for semi-supervised learning. Given an adjacency matrix and a few labelled vertices, unlabeled nodes can be described as a function of the labelled nodes based on the graph manifolds. In this work, our goal is not classification; instead, we model the centrality of the graph as a tool for ranking images. This is an extension of [7], in which image similarities are used to find a single most representative, or "canonical" image from image search results. Here, we use well understood methods for graph analysis based on PageRank, and provide a large-scale study of both the performance and computational costs of such system.

\subsection{Contribution of this work}

This paper makes three contributions:

1. We introduce a novel, simple, algorithm to rank images based on their visual similarities.

2. We introduce a system to re-rank current Google image search results. In particular, we demonstrate that for a large collection of queries, reliable similarity scores among images can be derived from a comparison of their local descriptors.

3. The scale of our experiment is the largest among the published works for content-based-image ranking of which we are aware. Basing our evaluation on the most commonly searched for object categories, we significantly improve image search results for queries that are of the most interest to a large set of people.

The remainder of the paper is organized as follows. Section 2 introduces the algorithm and describes the construction of the image-feature based visual similarity graph. Section 3 studies the performance on queries with homogeneous and heterogeneous visual categories. Section 4 presents the experiments conducted and an analysis of the findings. Section 5 concludes the paper.

\section{APPROACH \& ALGORITHM}

Given a graph with vertices and a set of weighted edges, we would like to measure the importance of each of the vertices. The cardinality of the vertices, or the sum of geodesic distance to the surrounding nodes are all variations of centrality measurement. Eigenvector Centrality provides a principled method to combine the "importance" of a vertex with those of its neighbors in ranking. For example, other factors being equal, a vertex closer to an "important" vertex should rank higher than others. As an example of a successful application of Eigenvector Centrality, PageRank [3] pre-computes a rank vector to estimate the importance for all of the webpages on the Web by analyzing the hyperlinks connecting web documents.

Eigenvector Centrality is defined as the principle Eigenvector of a square stochastic adjacency matrix, constructed from the weights of the edges in the graph. It has an intuitive Random Walk explanation: the ranking scores correspond to the likelihood of arriving in each of the vertices by traversing through the graph (with a random starting point), where the decision to take a particular path is defined by the weighted edges.

The premise of using these visual-hyperlinks for the basis of random walks is that if a user is viewing an image, other related (similar) images may also be of interest. In particular, if image $u$ has a visual-hyperlink to image $v$, then there is some probability that the user will jump from $u$ to $v$. Intuitively, images related to the query will have many other images pointing to them, and will therefore be visited often (as long as they are not an isolated and in a small clique). The images which are visited often are deemed important. Further, if we find that an image, $v$, is important and it links to an image $w$, it is casting its vote for $w$ 's importance and because $v$ is itself important, the vote should count more than a "non-important" vote.

Like page rank, the image rank (IR) is iteratively defined as the following:

$$
I R=S^{*} \times I R
$$

$S^{*}$ is the column normalized, symmetrical adjacency matrix $S$ where $S_{u, v}$ measures the visual similarity between image $u$ and $v$. Since we assume similarities are commutative, the similarity matrix $S$ is undirected. Repeatedly multiplying $I R$ by $S^{*}$ yields the dominant eigenvector of the matrix $S^{*}$. Although $I R$ has a fixed point solution, in practice it can be estimated more efficiently through iterative approaches.

The image rank converges only when matrix $S^{*}$ is aperiodic and irreducible. The former is generally true for the web, and the later usually requires a strongly connected graph, a property guaranteed in practice by introducing a damping factor $d$ into Equation 1. Given $n$ images, IR is defined as:

$$
I R=d S^{*} \times I R+(1-d) p, \quad \text { where } p=\left[\frac{1}{n}\right]_{n \times 1} .
$$

This is analogous to adding a complete set of weighted outgoing edges for all the vertices. Intuitively, this creates a small probability for a random walk to go to some other images in the graph, although it may not have been initially linked to the current image. $d>0.8$ is often chosen for practice; empirically, we have found the setting of $d$ to have relatively minor impact on the global ordering of the images.

\subsection{Features generation and representation}

A reliable measure of image similarity is crucial to good performance since this determines the underlying graph structure. Global features like color histograms and shape analysis, when used alone, are often too restrictive for the breadth of image types that need to be handled. For example, as shown in Figure 3, the search results for "Prius" often contains images taken from different perspectives, with different cameras, focal lengths, compositions and etc.

Compared with global features, local descriptors contain a richer set of image information and are relatively stable under different transformations and, to some degree, lighting variations. Examples of local features include Harris corners [6], Scale Invariant Feature Transform (SIFT) [10], Shape Context [2] and Spin Images [9] to name a few. Mikolajczyk et al. [11] presented a comparative study of various 

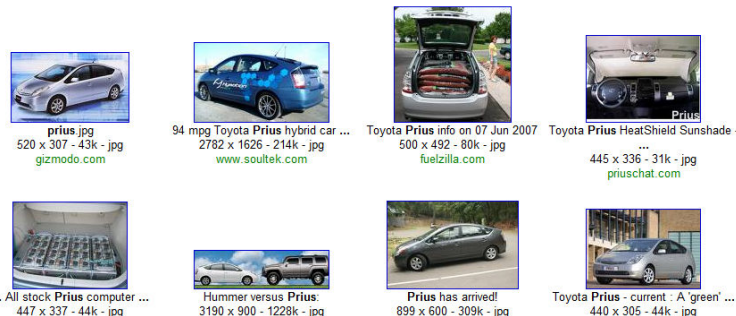

Hummer versus Prius
$3190 \times 900-1228 \mathrm{~K}-\mathrm{jpg}$
Wwww- pocinst. 0 .
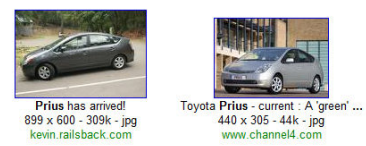

Figure 3: Similarity measurement must handle potential rotation, scale and perspective transformations.

descriptors, [18] [1] presented work on improving the their performance and computational efficiency. In this work, we use the SIFT features, with a Difference of Gaussian (DoG) interest point detector and orientation histogram feature representation as image features. Nonetheless, any of the local features could have been substituted.

We used a standard implementation of SIFT; for completeness, we give the specifics here. A DoG interest point detector builds a pyramid of scaled images by iteratively applying Gaussian filters to the original image. Adjacent Gaussian images are subtracted to create Difference of Gaussian images, from which the characteristic scale associated with each of the interest points can be estimated by finding the local extrema over the scale space. Given the DoG image pyramid, interest points located at the local extrema of $2 \mathrm{D}$ image space and scale space are selected. A gradient map is computed for the region around the interest point and then divided into a collection of subregions, in which an orientation histogram can be computed. The final descriptor is a 128 dimensional vector by concatenating $4 \times 4$ orientation histogram with 8 bins.

Given two images $u$ and $v$, and their corresponding descriptor vector, $D_{u}=\left(d_{u}^{1}, d_{u}^{2}, \ldots d_{u}^{m}\right)$ and $D_{v}=\left(d_{v}^{1}, d_{v}^{2}, \ldots d_{v}^{n}\right)$, we define the similarity between two images simply as the number interest points shared between two images divided by their average number of interest points.

\subsection{Query Dependent Ranking}

It is computationally infeasible to generate the similarity graph $S$ for the billions of images that are indexed by commercial search engines. One method to reduce the computational cost is to precluster web images based using metadata such as text, anchor text, similarity or connectivity of the web pages on which they were found, etc. For example, images associated with "Paris", "Eiffel Tower", "Arc de Triomphe" are more likely to share similar visual features than random images. To make the similarity computations more tractable, a different rank can be computed for each group of such images.

A practical method to obtain the initial set of candidates mentioned in the previous paragraph is to rely on the existing commercial search engine for the initial grouping of semantically similar images. For example, similar to [5], given the query "Eiffel Tower" we can extract the top- $N$ results returned, create the graph of visual similarity on the $N$ images, and compute the image rank only on this subset. In this instantiation, the approach is query dependent. In

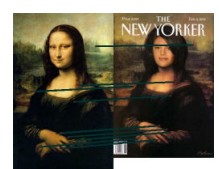

(a) A v.s. B

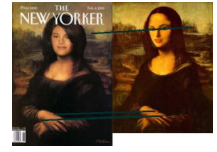

(d) B v.s. C

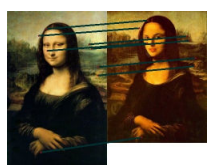

(b) A v.s. C

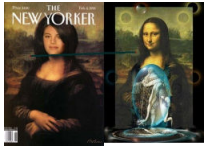

(e) B v.s. D

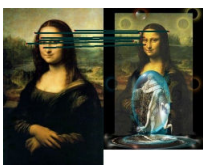

(c) A v.s. D

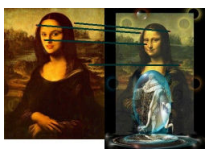

(f) C v.s. D
Figure 4: Since all the variations (B, C, D) are based on the original painting (A), A contains more matched local features than others.

the experiment section, we follow this procedure on 2000 of the most popular queries for Google Product Search.

\section{A FULL RETRIEVAL SYSTEM}

The goal of image-search engines is to retrieve image results that are relevant to the query and diverse enough to cover variations of visual or semantic concepts. Traditional search engines find relevant images largely by matching the text query with image metadata (i.e. anchor text, surrounding text). Since text information is often limited and can be inaccurate, many top ranked images may be irrelevant to the query. Further, without analyzing the content of the images, there is no reliable way to actively promote the diversity of the results. In this section, we will explain how the proposed approach can improve the relevancy and diversity of image search results.

\subsection{Queries with homogeneous visual concepts}

For queries that have homogeneous visual concepts (all images look somewhat alike) the proposed approach improves the relevance of the search results. This is achieved by identifying the vertices that are located at the "center" of weighted similarity graph. "Mona-lisa" is a good example of search query with a single homogeneous visual concept. Although there are many comical variations (i.e. "Bikini-lisa", "Monica-Lisa"), they are all based on the original painting. As shown in Figure 4, the original painting contains more matched local features than others, thus has the highest likelihood of visit by an user following these probabilistic visualhyperlinks. Figure 5 is generated from the top 1000 search results of "Mona-Lisa." The graph is very densely connected, but not surprisingly, the center of the images all correspond to the original version of the painting.

\subsection{Queries with heterogeneous visual concepts}

In the previous section we showed an example of improved performance with homogeneous visual concepts. In this section, we demonstrate it with queries that contain multiple visual concepts. Examples of such queries that are often given in information retrieval literature include "Jaguar" (car and animal) and "Apple" (computer and fruit). However, when considering images, many more queries also have multiple canonical answers; for example, the query "Nemo", shown earlier, has multiple good answers. In practice, we found that the approach is able to identify a relevant and diverse 


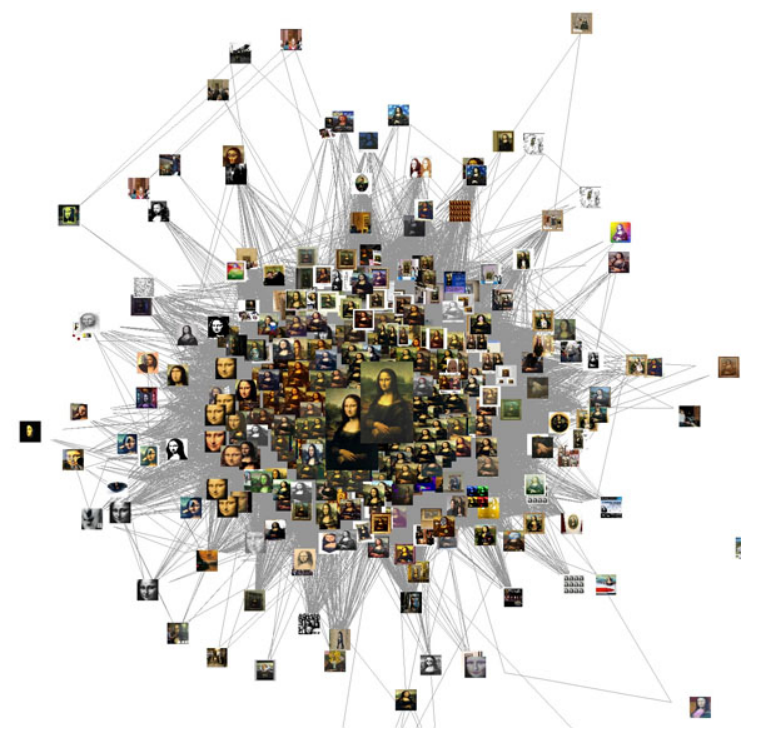

Figure 5: Similarity graph generated from the top 1000 search results of "Mona-Lisa." The largest two images contain the highest rank.

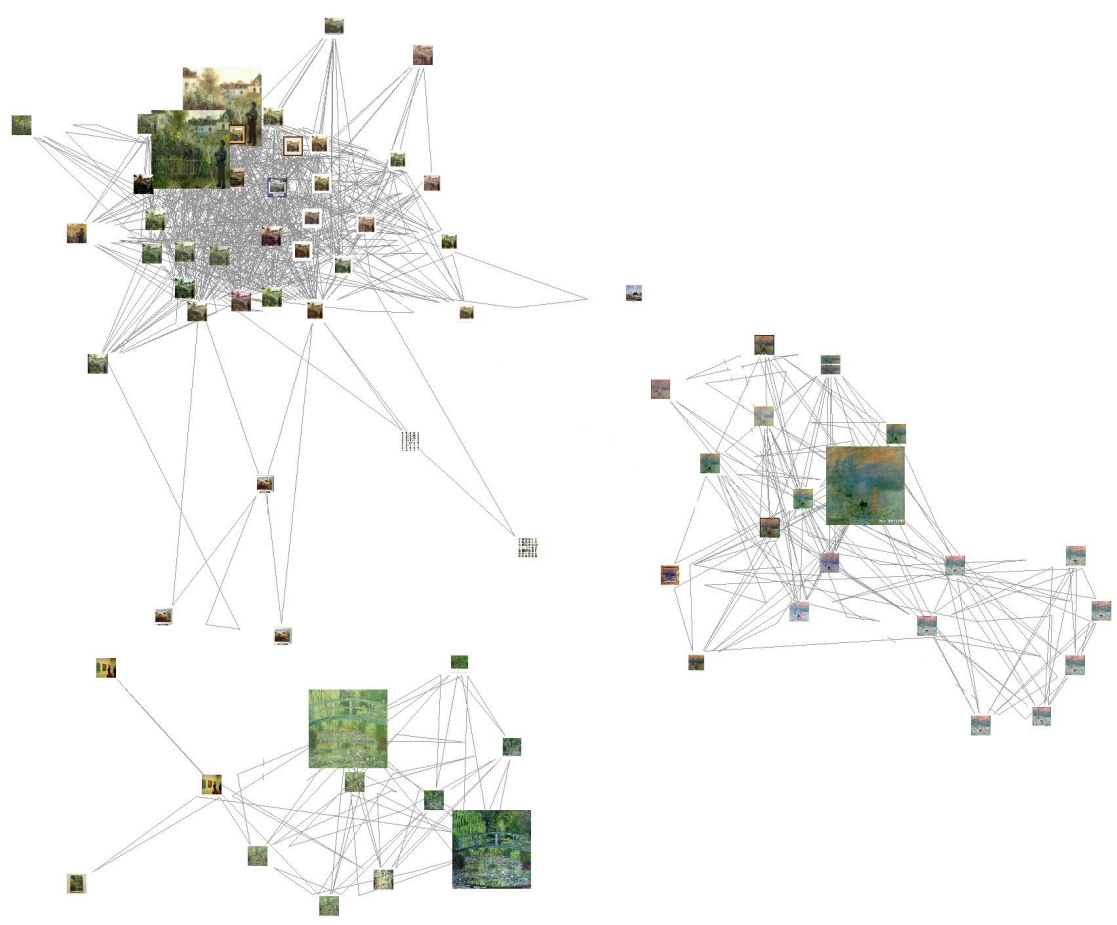

Figure 6: Top ten images selected from the 1000 search results of "Monet Paintings." By analyzing the link structure in the graph, note that highly relevant yet diverse set of images are found. Images include those by Monet and of Monet (by Renoir) 


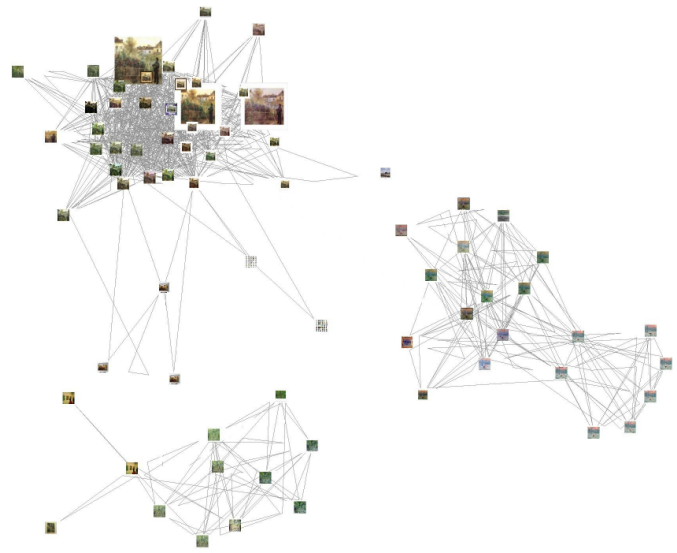

Figure 7: Alternative method of selecting images with the most "neighbors" tend to generate relevant but homogeneous set of images.

set of images as top ranking results; there is no apriori bias towards a fixed number of concepts or clusters.

A question that arises is whether simple heuristics could have been employed for analyzing the graph, rather than using a the Eigenvector approach used here. For example, a simple alternative is to select the high degree nodes in the graph, as this implicitly captures the notion of wellconnected images. However, this fails to identify the different distinctive visual concepts as shown in Figure 7. Since there are more close matches of "Monet Painting in His Garden at Argenteuil" by Renoir, they reinforce each other to form a strongly connected clique, and these are the only images returned.

\section{EXPERIMENTAL RESULTS}

To ensure that our algorithm works in practice, we conducted experiments with images collected directly from the web. In order to ensure that the results would make a significant impact in practice, we concentrated on the 2000 most popular product queries ${ }^{1}$ on Google (product search). These queries are popular in actual usage, and users have a strong expectations of the type of results each should return. Typical queries included "ipod", "xbox", "Picasso", "Fabreze", etc.

For each query, we extracted the top 1000 search results from Google Image Search on July 23rd, 2007, with the strict safe search filter. The similarity matrix is constructed by counting the number of matched local features for each pair of images after geometric validation normalized by the number of descriptors generated from each pairs of images.

We expect that Google's results will already be quite good, especially since the queries chosen are the most popular product queries for which many relevant web pages and images exist. Therefore, we would suggest a refinement to the ranking of the results when we are confident there is enough information to work correctly. A simple threshold was employed: if, in the set of 1000 images returned, fewer than $5 \%$ of the images had at least 1 connection, no modification was

\footnotetext{
${ }^{1}$ The most often queried keywords during a one month period.
}

suggested. In these cases, we assumed that the graph was too sparse to contain enough information. After this pruning, we concentrated on the approximately 1000 remaining queries.

It is challenging to quantify the quality of (or difference of performance) of sets of image search results for several reasons. First, and foremost, user preference to an image is heavily influenced by a user's personal tastes and biases. Second, asking the user to compare the quality of a set of images is a difficult, and often a time consuming task. For example, an evaluator may have trouble choosing between group A, containing five relevant but mediocre images, and group B, that is mixed with both great and bad results. Finally, assessing the differences in ranking (when many of the images between two rankings being compared are the same) is error-prone and imprecise, at best. Perhaps the most principled way to approach this task is to build a global ranking based on pairwise comparisons. However, this process requires significant amount of user input, and is not feasible for large numbers of queries.

To accurately study the performance, subject to practical constraints, we devised two evaluation strategies. Together, they offer a comprehensive comparison of two ranking algorithms, especially with respect to how the rankings will be used in practice.

\subsection{Minimizing Irrelevant Images}

This study is designed to study a conservative version of "relevancy" of our ranking results. For this experiment, we mixed the top 10 selected images using our approach with the top 10 image from Google, removed the duplicates, and presented them to the user. We asked the user: "Which of the image(s) are the least relevant to the query?" For this experiment, more than 150 volunteer participants were chosen, and were asked this question on a set of randomly chosen 50 queries selected from the top-query set. There was no requirement on the number of images that they marked.

There are several interesting points to note about this study. First, it does not ask the user to simply mark relevant images; the reason for this is that we wanted to avoid a heavy bias to a user's own personal expectation (i.e. when 
Table 1: "Irrelevant" images per product query

\begin{tabular}{l|c|c|} 
& Image Rank & Google \\
\hline Among top 10 results & 0.47 & 2.82 \\
\hline Among top 5 results & 0.30 & 1.31 \\
\hline Among top 3 results & 0.20 & 0.81 \\
\hline
\end{tabular}

querying "Apple" did they want the fruit or the computer?). Second, we did not ask the users to compare two sets; since, as mentioned earlier, this is an arduous task. Instead, the user was asked to examine each image individually. Third, the user was given no indication of ranking; thereby alleviating the burden of analyzing image ordering.

It is also worth noting that minimizing the number of irrelevant images is important in real-world usage scenarios beyond "traditional" image search. In many uses, we need to select a very small set (1-3) of images to show from potentially millions of images. Unlike ranking, the goal is not to reorder the full set of images, but to select only the "best" ones to show. Two concrete usage cases for this are: 1 . Google product search: only a single image is shown for each product returned in response to a product query; shown in Figure 8(a). 2. Mixed-Result-Type Search: to indicate that image results are available when a user performs a web (webpage) query, a small set of representative images may also be shown to entice the user to try the image search as shown in Figure 8(b). In both of these examples, it is paramount that the user is not shown irrelevant, off-topic, images. Both of these scenarios benefit from procedures that perform well on this experimental setup.

We measured the results at three settings: the number of irrelevant images in the top-10, top-5, and top-3 images returned by each of the algorithms. Table 1 contains the comparison results. Among the top 10 images, we produced an average of 0.47 irrelevant results, this is compared with 2.82 by Google; this represents an $83 \%$ drop in irrelevant images. When looking at the top-3 images, the number of irrelevant images dropped to 0.2 , while Google dropped to 0.81 .

In terms of overall performance on queries, the proposed approach contains less irrelevant images than Google for 762 queries. In only 70 queries did Google's standard image search produce better results. In the remaining 202 queries, both approaches tied (in the majority of these, there were no irrelevant images). Figure 9 shows examples of top ranking results for a collection of queries. Aside from the generally intuitive results shown in Figure 9, an interesting result is shown for the query "Picasso Paintings"; not only are all the images by Picasso, one of his most famous, "Guernica", was selected first.

To present a complete analysis, we describe two cases that did not perform as expected. Our approach sometimes fails to retrieve relevant images as shown in Figure 10. The first three images are the logos of the company which manufactured the product being searched for. Although the logo is somewhat related to the query, the evaluators did not regard them as relevant to the specific product for which they were searching. The inflated logo score occurs for two reasons. First, many product images contains the company logos; either within the product itself or in addition to the product. In fact, extra care is often given to make sure that the logos are clearly visible, prominent, and uniform in appearance.

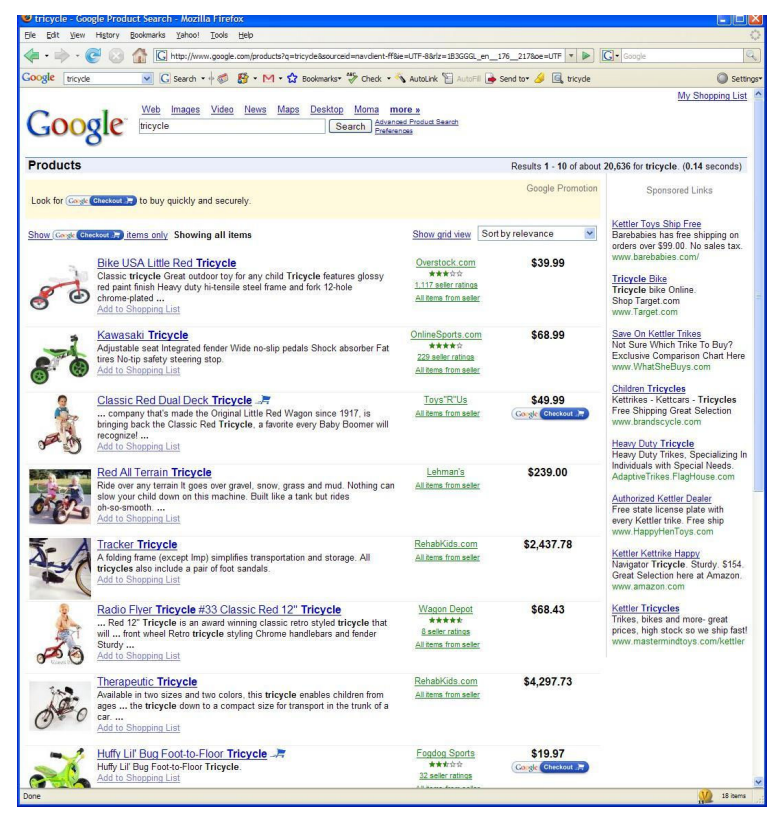

(a) Google product search

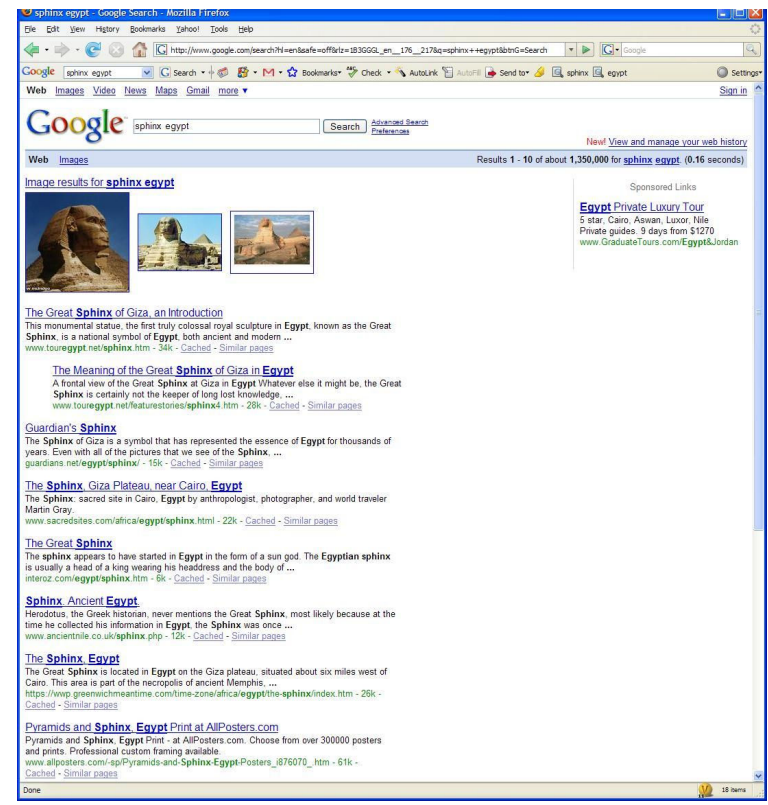

(b) Mixed-Result-Type Search

Figure 8: In many uses, we need to select a very small set (1-3) of images to show from potentially millions of images. Unlike ranking, the goal is not to reorder the full set of images, but to select only the "best" ones to show. 


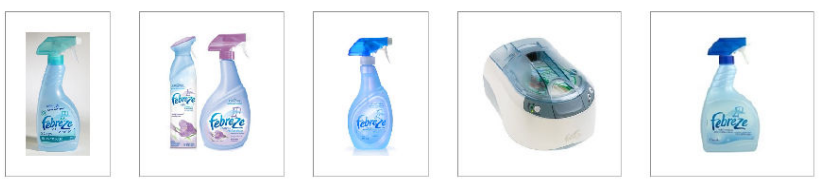

(a) Fabreze

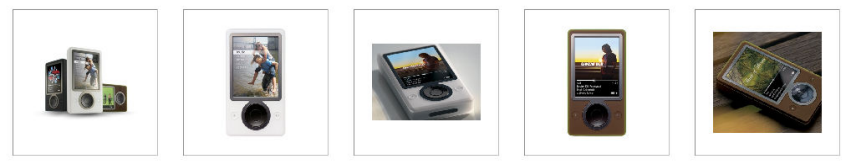

(b) Microsoft Zune

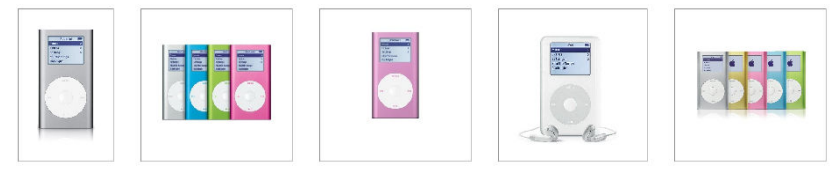

(c) Ipod Mini

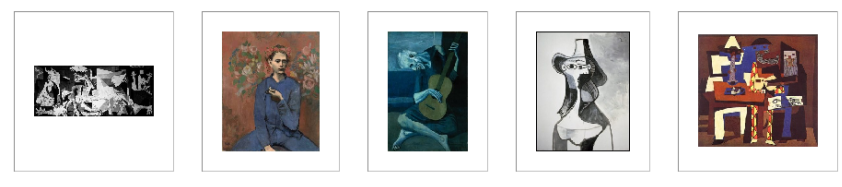

(d) Picasso Paintings

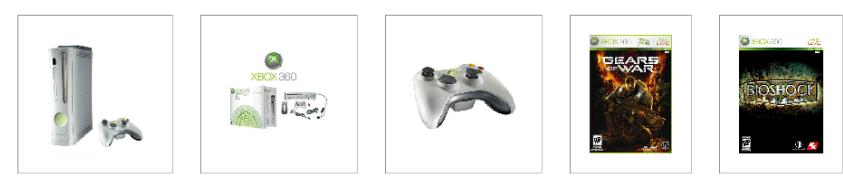

(e) Xbox Games

Figure 9: An example of top product images selected.
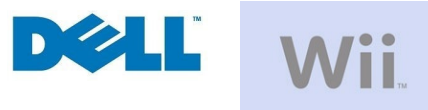

(a) dell com- (b) nintendo puter

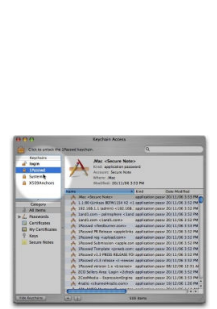

(d) keychain wii system

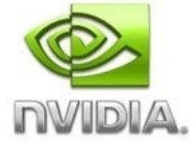

(c) 8800 Ultra

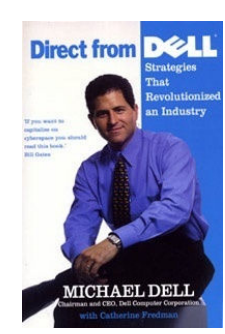

(e) ps 2 network (f) dell comadapter puter
Figure 10: The particular local descriptors used provided a bias to the types of patterns found. These images, selected by our approach, received the most "irrelevant" votes from the users for the queries shown.
Second, logos often contain distinctive patterns that provides a rich set of local descriptors that are particularly well suited to SIFT-like feature extraction.

A second, but less common, failure case is when screenshots of web pages are saved as images. Many of these images include browser panels or Microsoft Window's control panels that are consistent across many images. It is suspected that these mismatches can easily be filtered by employing other sources of quality scores or measuring distinctiveness of the features not only within queries but also across queries; in a manner similar to using TF-IDF [14] weighting in textual relevancy.

\subsection{Click Study}

Results from the previous experiment show that we can effectively decrease the number of irrelevant images in the search results. However, user satisfaction is not purely a function of relevance; for example, numerous other factors such as diversity of the selected images must also be considered. Assuming the users usually click on the images they are interested in, an effective way to measure search quality is to analyze the total number of "clicks" each image receives.

We collected clicks for the top 40 images (first two pages) presented by the Google search results on 130 common product queries. For the top-1000 images for each of the 130 queries, we rerank them according to the approach described. To determine if the ranking would improve performance, we examine the number of clicks each method received from only the top-20 images (these are the images that would be displayed in the first page of results of Google's image search). The hope is that by reordering the top-40 results, the best images will move to the top and would be displayed on the first page of results. If we are successful, then the number of clicks for the top-20 results under reordering will exceed the number of clicks for the top-20 under the default ordering.

It is important to note that this evaluation contains an extremely severe bias that favors the default ordering. The ground-truth of clicks an image receives is a function not only of the relevance to a query and quality of the image, but of the position in which it is displayed. For example, it is often the case that a mediocre image from the top of the first page will receive more clicks than a high quality image from the second page (default ranking 21-40). If we are able to outperform the existing Google Image search in this experiment, we can expect a much greater improvement in deployment.

When examined over the set of 130 product queries, the images selected by our approach to be in the top-20 would have received approximately $17.5 \%$ more clicks than those in the default ranking. This improvement was achieved despite the positional bias that strongly favored the default rankings.

\section{CONCLUSIONS}

The algorithms presented in this paper describe a simple mechanism to incorporate the advancements made in using link and network analysis for web-document search into image search. Although no links explicitly exist in the image search graph, we demonstrated an effective method to infer a graph in which the images could be embedded. The result was an approach that was able to outperform the default Google ranking on the vast majority of queries tried. 
Importantly, the ability to reduce the number of irrelevant images shown is extremely important not only for the task of image ranking for image retrieval applications, but also for applications in which only a tiny set of images must be selected from a very large set of candidates.

Interestingly, by replacing user-created hyperlinks with automatically inferred "visual-hyperlinks", the proposed approach seems to deviate from a crucial source of information that makes PageRank successful: the large number of manually created links on a diverse set of pages. However, a significant amount of the human-coded information is recaptured through two mechanisms. First, by making the approach query dependent (by selecting the initial set of images from search engine answers), human knowledge, in terms of linking relevant images to webpages, is directly introduced into the system, since it the links on the pages are used by Google for their current ranking. Second, we implicitly rely on the intelligence of crowds: the image similarity graph is generated based on the common features between images. Those images that capture the common themes from many of the other images are those that will have higher rank.

The categories of queries addressed, products, lends itself well to the type of local feature detectors that we employed to generate the underlying graph. One of the strengths of the approach described in this paper is the ability to customize the similarity function based on the expected distribution of queries. Unlike classifier based methods [5] [13] that construct a single mapping from image features to ranking, we rely only on the inferred similarities, not the features themselves. Similarity measurements can be constructed through numerous techniques; and their construction is independent of the image relevance assessment. For example, images related to people and celebrities may rely on face recognition/similarity, images related products may use local descriptors, other images such as landscapes, may more heavily rely on color information, etc. Additionally, within this framework, context-free signals, like user-generated covisitation [16], can be used in combination with image features to approximate the visual similarity of images.

Inferring visual similarity graphs and finding PageRanklike scores opens a number of opportunities for future research. Two that we are currently exploring are (1) determining the performance of the system under adversarial conditions. For example, it may be possible to bias the search results simply by putting many duplicate images into our index. We need to explore the performance of our algorithm under such conditions. 2) the role of duplicate and nearduplicate images must be carefully studied, both in terms of the potential for biasing our approach, and also in terms of transition probabilities. It may be unlikely that a user who has visited one image will want to visit another that is a close or an exact duplicate. We hope to model this explicitly in the transition probabilities.

\section{REFERENCES}

[1] H. Bay, T. Tuytelaars, and L. V. Gool. Surf: Speeded up robust features. In Proc. 9th European Conference on Computer Vision (ECCV), pages 404-417, 2006.

[2] S. Belongie, J. Malik, and J. Puzicha. Shape matching and object recognition using shape contexts. IEEE Transactions on Pattern Analysis and Machine Intelligence (PAMI), 24(24):509-522, 2002.
[3] S. Brin and L. Page. The anatomy of a large-scale hypertextual Web search engine. Computer Networks and ISDN Systems, 30(1-7):107-117, 1998.

[4] R. Diestel. Graph Theory. Springer, New York, NY, 2005.

[5] R. Fergus, P. Perona, and A. Zisserman. A visual category filter for google images. In Proc. 8th European Conference on Computer Vision (ECCV), pages 242-256, 2004.

[6] C. Harris and M. Stephens. A combined corner and edge detector. In Proc. 4th Alvey Vision Conference, pages 147-151, 1988.

[7] Y. Jing, S. Baluja, and H. Rowley. Canonical image selection from the web. In Proc. 6th International Conference on Image and Video Retrieval (CIVR), 2007.

[8] R. I. Kondor and J. Lafferty. Diffusion kernels on graphs and other discrete structures. In Proc. 19th International Conference on Machine Learning (ICML), 2002.

[9] S. Lazebnik, C. Schmid, and J. Ponce. A sparse texture representation using affine-invariant regions. In Proc. Conference on Computer Vision and Pattern Recognition (CVPR), pages 319-324, 2003.

[10] D. G. Lowe. Distinctive Image Features from Scale-Invariant Keypoints. International Journal of Computer Vision, 60(2):91-110, 2004.

[11] K. Mikolajczyk and C. Schmid. A performance evaluation of local descriptors. IEEE Transaction on Pattern Analysis and Machine Intelligence, 27(10):1615-1630, 2005.

[12] D. Nistér and H. Stewénius. Scalable recognition with a vocabulary tree. In Proc. Conference on Computer Vision and Pattern Recognition (CVPR), volume 2, pages 2161-2168, 2006.

[13] G. Park, Y. Baek, and H. Lee. Majority based ranking approach in web image retrieval. Lecture notes in computer science, pages 111-120, 2003.

[14] G. Salton and M. J. McGill. Introduction to Modern Information Retrieval. McGraw-Hill Book Co., New York, NY, 1983.

[15] H. Schneiderman. Learning a restricted Bayesian network for object detection. In Proc. Conference on Computer Vision and Pattern Recognition (CVPR), pages 639-646, 2004.

[16] S. Uchihashi and T. Kanade. Content-free image retrieval by combinations of keywords and user feedbacks. In Proc. 5th International Conference on Image and Video Retrieval (CIVR), pages 650-659, 2005.

[17] P. Viola and M. Jones. Robust real-time object detection. International Journal of Computer Vision, 57:137-154, May 2004.

[18] S. Winder and M. Brown. Learning local image descriptors. In Prof. Conference on Computer Vision and Pattern Recognition (CVPR), pages 1-8, 2007.

[19] X. Zhu and Z. Ghahramani. Learning from labeled and unlabeled data with label propagation, 2002. 